\title{
Demonstration of negative signal delay with short-duration transient pulse
}

\author{
Blaise Ravelo \\ IRSEEM, EA 4353, \\ at Graduate School of Engineering ESIGELEC, \\ Av. Galilée, BP 10024, 76801 Saint Etienne du Rouvray Cedex, France. \\ Tel: +33 (0)2 32915971 , \\ Fax: +33(0)232915859. \\ E-mail: blaise.ravelo@yahoo.fr,blaise.ravelo@esigelec.fr
}

\begin{abstract}
This paper introduces theoretic and experimental analyses of short-duration pulse propagation through a negative group delay (NGD) circuit. The basic analysis method of this electronic circuit operating in baseband and microwave frequencies is investigated. Then, its electrical fundamental characteristics vis-à-vis transient signals are developed. To validate the theoretic concept, planar hybrid devices with one- and two-stage NGD cells were designed, simulated, fabricated and tested. Transient analyses with ultra-wide band (UWB) pulse signals with different widths are realized. Then, experimental results in good agreement with the theoretical predictions were observed. Consequently, group delay going down under $\mathbf{- 2 . 5}$ ns is evidenced in baseband frequency up to $63 \mathrm{MHz}$ with one-stage NGD cell. In time-domain, a Gaussian pulse in advance of about $t_{0}=-1.5 \mathrm{~ns}$ or $20 \%$ of its half-height time-width was measured. This corresponds to a negative group velocity of about $v_{g}=L / t_{0}=-0.13 c$ ( $\mathrm{L}$ is the physical length of the tested device and $c$ is light speed in the vacuum). More significant NGD value over 100 MHz-bandwidth is stated with two-stage NGD cells. This results a Gaussian pulse peak advance of about -5 ns (raising a group velocity of about $v_{g}=-0.12 c$ ) or $31 \%$ of its halfheight time-width. Finally, some potential applications based on the NGD function are discussed.
\end{abstract}

Keywords: Negative group delay (NGD), time advance, transient short-pulse, UWB application.

\section{INTRODUCTION}

In the early of the twentieth century, Lamb [1] and Schuster [2] predicted the existence of the anomalous dispersion where the refractive index, $n(\omega)$ is inversely proportional to the angular frequency band, $\omega$. The study of this phenomenon attracted numerous physicists. Notably, by analyzing the propagation of electromagnetic (EM) wave modulating a carrier frequency equal to the resonance frequency of the atomic vapour medium:

$$
\omega_{p}=2 e \sqrt{\frac{\pi N}{m}},
$$

having mass, $m$, charge, $e$ and with unit volume density, $N$, Sommerfeld and Brillouin [3-8], demonstrated theoretically the possibility to obtain a negative group velocity $(\mathrm{NGV})$ around this resonant frequency via the formula:

$$
v_{g}(\omega)=\frac{c}{\Re e\left[n(\omega)+\omega \cdot \frac{\partial n(\omega)}{\partial \omega}\right]},
$$


where $c$ is the light speed in the vacuum. It was shown in different physical domains [9-16] that in this case, the causality principle is respected. Due to the constructive and destructive interferences, it was verified theoretically and experimentally [10][17-22] that through this NGV medium, the output pulse peak can be detected in time advance of the input one, if the input pulse energy spectrum is limited in the NGV frequency band. In this case, it was emphasized that the pulse distortion can be neglected [19]. So, for an EM medium having a physical length, $L$, when $v_{g}<0$, the pulse transit time given by the group delay, $\tau=L / v_{g}$ is also naturally negative.

In 1990s, this negative group delay (NGD) phenomenon was also confirmed with several electronic experiments [2231]. Firstly, by using an operational amplifier in negative feedback loop with passive networks based on $\mathrm{R}, \mathrm{L}$ and $\mathrm{C}$, Chiao and his co-workers from the University of Berkeley proposed a topology of active circuit analogue to the resonant atomic system with negative refractive group index [22-24]. So, they have evidenced in time domain, an output Gaussian pulse peak in advance of about some hundreds of ms compared to the corresponding input. They pointed out also that this NGD phenomenon does not forbid the causality [23-25]. To characterize this counterintuitive phenomenon, it is more general to use the group delay concept instead of the group velocity because in the electronic devices, the lumped component physical length is meaningless. In addition, for a system having a transfer function, $H(j \omega)$, the group delay can be defined as:

$$
\tau(\omega)=-\frac{\partial \angle H(j \omega)}{\partial \omega} .
$$

Therefore, based on this topology, Kitano's group [26-27] have evidenced this NGD phenomenon, by demonstrating the possibility to light on the LED connected at the output of an NGD circuit before the input one. Furthermore, as reported in [28], the authors confirmed a time domain advance of complex audio wave operating at hundreds of kHz. In 2002, Chiao and his team [29] proposed to use this NGD circuit to cancel out the propagation delays of interconnections and bus links in the microelectronic devices. As reported in [29], the authors demonstrated experimentally the elimination of propagation delays from RC time constants when considering a square wave data of $2.5 \mathrm{kbits} / \mathrm{s}-\mathrm{rate}$. Nevertheless, the main roadblock of this application for the numerous high speed submicron systems that such a topology is limited to some MHz. Hence, passive microwave NGD lumped circuits, capable to operate up to GHz, have been proposed by the groups of Lucyszyn [30], Broomfield [31] and of Eleftheriades [32-33]. But, due to the excessive inherent losses and the narrow bandwidth systematically associated to the generation of significant NGD value, this circuit is inoperable in many electronic systems. To pass these limits, recently, a topology of microwave active circuit (Fig. 1) capable to generate NGD with losses compensation have been developed in [34-36] using a field effect transistor (FET).

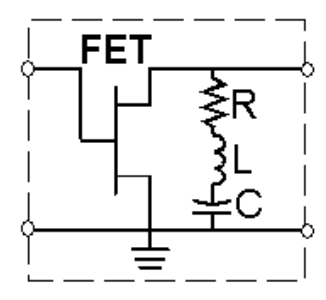

Fig. 1. NGD microwave active circuit.

It was established that at the resonance, $\omega_{0}=1 / \sqrt{L C}$ of this cell, the group delay expressed in (4) is always negative for all values of the parameters, $R, L$ and $R_{d s}$, and it does not depend on the capacitance, $C$. 


$$
\tau\left(\omega_{0}\right)=\frac{-2 L \cdot R_{d s}}{R \cdot\left(R+R_{d s}\right)} .
$$

By cons, at very low frequency, the group delay of this circuit is always positive. It means that this NGD circuit is mainly intended to the applications based on the modulated signals having carrier up to some GHz. This limit is the source of the motivation to study the another topology of NGD active circuit composed of an RL series network in feedback with an FET depicted in Figs. 2 [37-41]. According to the characteristics of the employed FET, it was reported that this circuit can exhibit an ultra-wide band (UWB) NGD from DC up to some hundreds MHz without losses [38]. As application, this NGD circuit was employed to reduce the delay and to compensate losses induced by high rate submicron interconnects lines have been developed in [38-43] thanks to the technique introduced by Chiao's group in [29]. In addition, microwave application for the design of independent-frequency phase shifter is also introduced in [4445].

For this reason, deeper investigation on the UWB NGD active circuit is devoted in this paper. The novelty of the latter is the detailed development of the main theoretic characteristics and smart properties of the NGD topology depicted in Fig. 2 and the time-domain demonstration of negative signal delay by using an ultra-short duration pulse. To do so, in Section 2, frequency- and time-domain investigations based on the examination of the under study circuit transfer function is presented. Section 3 is dealing with the realized experimental results enabling the validation of the established theoretic concept about the NGD phenomenon and the discussions about some potential applications. Finally, concluding remarks of this work is drawn in the last section.

\section{THEORY}

The main purpose of this section is the development of the fundamental theory on the UWB NGD active circuit schematized in Fig. 2. This circuit consists of a series resistance, $R$, and inductance, $L$ in feedback with an FET.

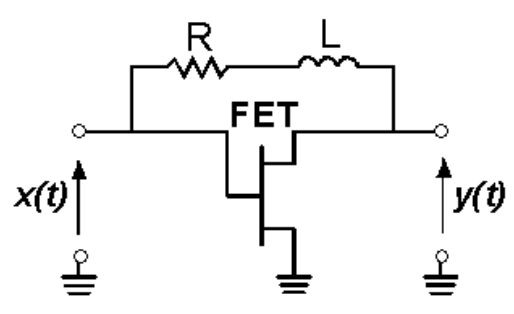

Fig. 2. Basic cell of the NGD active circuit.

For the simplification of analytical expressions, the employed FET is modeled by a voltage-controlled current source with a transconductance, $g_{m}$, in cascade with the drain-source resistor, $R_{d s}$.

\subsection{Generality on the understudied NGD cell}

By means of Laplace transform, it was established that the electronic cell depicted in Fig. 2 presents a voltage transfer function written as [38]:

$$
H(s)=\frac{Y(s)}{X(s)}=\frac{R_{d s}\left(1-g_{m} R-g_{m} L \cdot s\right)}{R_{d s}+R+L \cdot s} .
$$


By taking $s=j \omega$ (with $j=\sqrt{-1}$ ), this expression implies the magnitude and group delay frequency responses given by:

$$
\begin{aligned}
& |H(j \omega)|=\sqrt{\frac{\left(R+R_{d s}\right)^{2}\left[\left(1-g_{m} R\right)^{2}+\left(g_{m} L \omega\right)^{2}\right]}{\left(1-g_{m} R\right)^{2}\left[\left(R+R_{d s}\right)^{2}+(L \omega)^{2}\right]}}, \\
& \tau(\omega)=\frac{L\left(1+g_{m} R_{d s}\right)\left[\left(R+R_{d s}\right)\left(1-g_{m} R\right)+g_{m}(L \omega)^{2}\right]}{\left[\left(g_{m} R-1\right)^{2}+\left(g_{m} L \omega\right)^{2}\right]\left[\left(R+R_{d s}\right)^{2}+(L \omega)^{2}\right]} .
\end{aligned}
$$

Therefore, at very low frequency or $\omega \approx 0$, the group delay is equal to:

$$
\tau(\omega \approx 0)=\frac{L\left(1+g_{m} R_{d s}\right)}{\left(1-g_{m} R\right)\left(R_{d s}+R\right)} .
$$

This last relation enables to write the following condition of the NGD existence at $\omega \approx 0$ :

$$
1-g_{m} \cdot R<0 \Leftrightarrow R>1 / g_{m} .
$$

Thus, under this condition, the transfer function magnitude expressed in (6) becomes:

$$
|H(\omega \approx 0)|=\frac{R_{d s}\left(g_{m} R-1\right)}{R_{d s}+R} .
$$

So, one realizes a gain $|H(\omega \approx 0)|>1$ when the following inequality is verified:

$$
R>\frac{2 R_{d s}}{g_{m} R_{d s}-1} .
$$

\subsection{NGD cell synthesis}

Similar to all classical electronic functions, this synthesis is aimed at the determination of the NGD cell elements, $R$ and $L$ in function of the desired gain and the desired group delay respectively, denoted $H_{0}$ and $\tau_{0}$ at very low frequency. Knowing the FET characteristics, $g_{m}$ and $R_{d s}$, and by inverting the equations $\tau(\omega \approx 0)=\tau_{0}$ and $|H(\omega \approx 0)|=H_{0}$, one establishes the following synthesis relations:

$$
\begin{aligned}
& R=R_{d s} \frac{H_{0}+1}{g_{m} R_{d s}-H_{0}}, \\
& L=\frac{-\tau_{0} H_{0} R_{d s}\left(g_{m} R_{d s}+1\right)}{\left(g_{m} R_{d s}-H_{0}\right)^{2}} .
\end{aligned}
$$

These expressions permit to plot the synthesis charts presented respectively in Fig. 3 and in Figs. 4 for the determination of $R$ and $L$ versus the gain, $H_{0 \mathrm{~dB}}$.

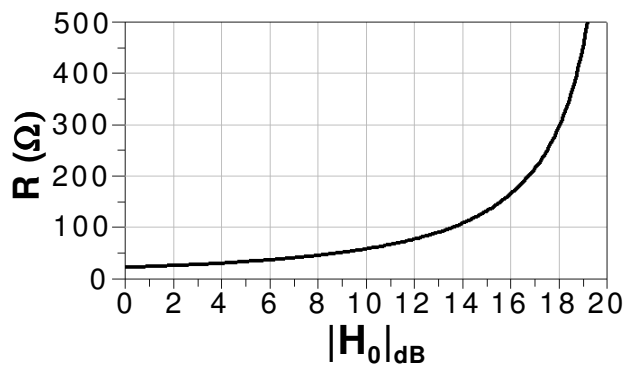

Fig. 3. $R$-synthesis curve versus $H_{0 d B}$ for $g_{m}=98.14 \mathrm{mS}$ and $R_{d s}=116.8 \Omega$. 
As explained in Fig. 3, the $R$-value is proportional to $H_{0}$ and it does not depend to $\tau_{0}$. Hence, as highlighted in Figs. 4 , the value of $L$ increases with $\left|\tau_{0}\right|$.
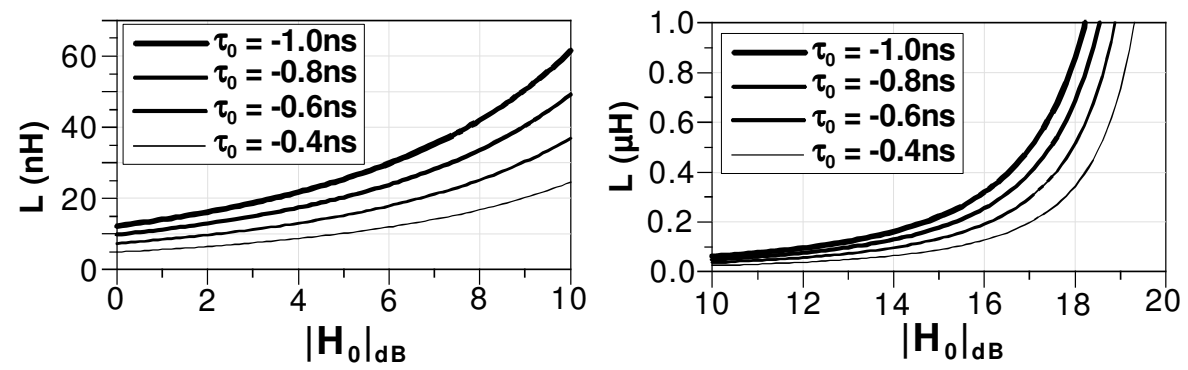

Fig. 4. $L$-synthesis charts versus $\tau_{0}$ (from $-1.0 \mathrm{~ns}$ to $-0.4 \mathrm{~ns}$ step $0.2 \mathrm{~ns}$ ) and $H_{0 d B}((\mathrm{a})$ from 0 to $10 \mathrm{~dB}$ and (b) from 10 to $20 \mathrm{~dB}$ ) for $g_{m}=98.14 \mathrm{mS}$ and $R_{d s}=116.8 \Omega$.

It is worth noting also that this inductance value remains positive because of the negative value of the group delay, $\tau_{0}$ which is annihilated by the sign minus in formula (13). During the synthesis, it is important to keep in mind that the maximal attainable gain for a given FET having characteristics, $g_{m}$ and $R_{d s}$ is equal to $H_{m}=g_{m} \cdot R_{d s}$. Substituting the $R$ - and $L$-synthesis relations expressed in (12) and (13) into equation (5), one obtains the following expression of the isochrones transmittance, $H(j \omega)$ in function of, $H_{0}$ and $\tau_{0}$ :

$$
H(s)=H_{0} \frac{H_{0}-H_{m}+\tau_{0} H_{m} s}{H_{0}-H_{m}+\tau_{0} H_{0} s} \Rightarrow H(j \omega)=H_{0} \frac{H_{0}-H_{m}+j \omega \tau_{0} H_{m}}{H_{0}-H_{m}+j \omega \tau_{0} H_{0}} .
$$

In that case, $\tau_{0}$ should be chosen to be negative. From these expressions, the particular properties of the understudy NGD cell can be stated.

\subsection{NGD cell properties}

First of all, it is worth underlining that the group delay expressed in (7) is negative when its numerator $\left(R+R_{d s}\right)\left(1-g_{m} R\right)+g_{m}(L \omega)^{2}$ is negative. It means that, this cell exhibits an NGD in the base band frequency delimited by $\omega_{c}=2 \pi f_{c}$. This NGD cut-off angular frequency, which is also the root of the equation, $\tau\left(\omega_{c}\right)=0$ is written as:

$$
\omega_{c}=\frac{\sqrt{\left(R+R_{d s}\right)\left(R-1 / g_{m}\right)}}{L} .
$$

Substituting the $R$ - and $L$-expressions written in (12) and (13) into (15), one can express this cut-off frequency in function of the desired gain, $H_{0}$ and group delay, $\tau_{0}$ :

$$
\omega_{c}=\frac{\left(H_{m}-H_{0}\right)}{\tau_{0} \sqrt{H_{m} H_{0}\left(H_{m}+1\right)}} .
$$

This formula enables to write the relation between the product between the NGD, $\tau_{0}$ and the cut-off frequency, $\omega_{c}$ according to the used FET maximal gain, $H_{m}$ :

$$
\left|\omega_{c} \tau_{0}\right|=\frac{H_{m}-H_{0}}{\sqrt{H_{m} H_{0}\left(H_{m}+1\right)}} .
$$


From where is established the chart plotted in Fig. 5 which illustrates the variation of this product in function of the desired gain, $H_{0}$.

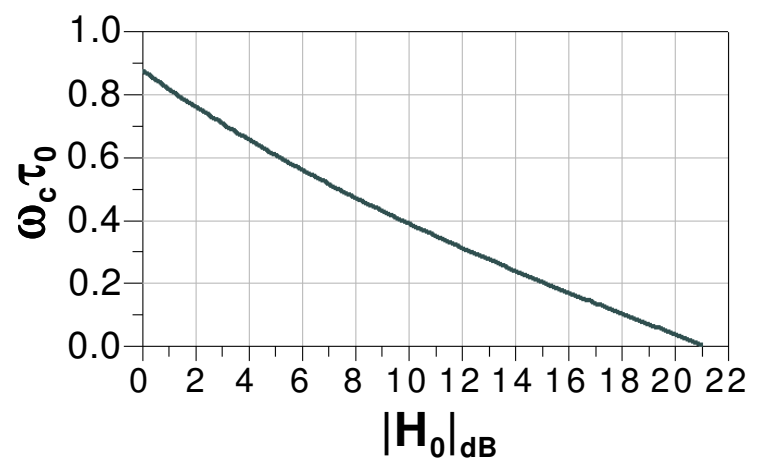

Fig. 5. Product of the NGD cut-off frequency and maximal absolute value $\left|\omega_{c} \tau_{0}\right|$ versus $\boldsymbol{H}_{0 d B}$

$$
\left(g_{m}=98.14 \mathrm{mS} \text { and } R_{d s}=116.8 \Omega\right) \text {. }
$$

This graph explains clearly that this cut-off frequency, $\omega_{c}$ is inversely proportional to the group delay absolute value, $|\tau(0)|$. One demonstrates also that the surface quantity, $S(\tau<0)$ limited by the group delay line, $\tau(\omega)$, the $X$-axis and the $Y$-axis as depicted by Fig. 6 is given by:

$$
S(\tau<0)=\arctan \left[\frac{\left(g_{m} R+1\right) \sqrt{\left(R+R_{d s}\right)\left(g_{m} R-1\right)}}{2\left[g_{m} R\left(R+R_{d s}\right)-R-R_{d s}\right] \sqrt{g_{m}}}\right]-\pi .
$$

It can be seen clearly that this quantity does not depend to $L$, then it is also obviously independent to the group delay value, $|\tau(0)|$. However, this surface increases when the resistance value, $R$ decreases and inversely.

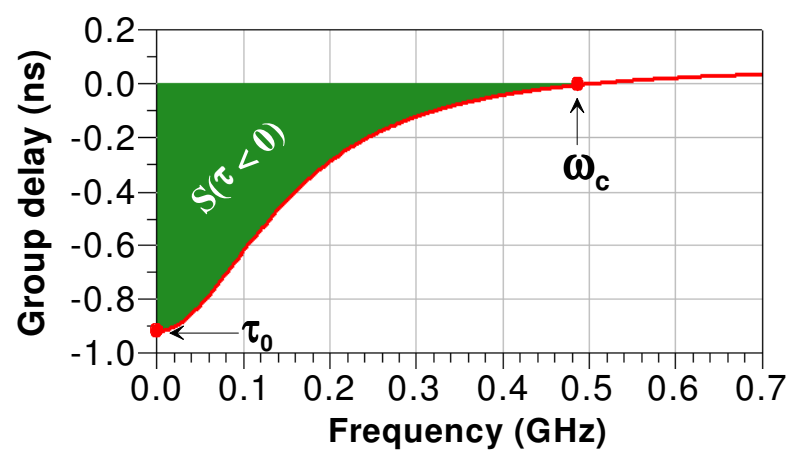

Fig. 6. Group delay versus Frequency of the circuit for $H_{0 d B}=0 \mathrm{~dB}, g_{m}=98.14 \mathrm{mS}$ and $R_{d s}=116.8 \Omega$.

This finding is highlighted by the example of the surface plot represented in Fig. 6 which shows the group delay, $\tau\left(f, \tau_{0}\right)$ in function of the frequency, $f=\omega /(2 \pi)$ and the group delay, $\tau_{0}$ for $H_{0 d B}$ which is here, taken equal to $0 \mathrm{~dB}$. In order to interpret more explicitly the theoretic concept on the NGD effect induced by the under study topology, timedomain analysis is proposed in the next subsection. 


\subsection{Time-domain investigation of NGD cell: analysis of Gaussian response}

Due to the drain-source current direction of the FET, the output voltage, $y(t)$ indicated in Figs. 2 is naturally in opposite of the input one, $x(t)$. Then, to highlight the signal integrity conservation, the shapes of $x(t)$ and the opposite of $y(t)$, denoted, $-y(t)$ are compared. Along this study, the normalized input, $x(t)$ is assumed as a Gaussian pulse expressed as:

$$
x(t)=e^{-\frac{\left(t-t_{0}\right)^{2}}{2 \Delta T^{2}}}
$$

with standard deviation, $\Delta T$ and centered at $t_{0}$ in order to evidence the time advance phenomenon. By considering the transfer function expressed in (15) and the Fourier transform expressed in (A-1), one obtains the corresponding output mathematically written as $Y(j \omega)=H(j \omega) \cdot X(j \omega)$ which implies the results given in (A-2). Knowing that $H^{\prime}(0)=\varphi(0)=\varphi^{\prime \prime}(0)=0$, and:

$$
H^{\prime \prime}(0)=\frac{H_{0} \tau_{0}^{2}\left(H_{m}+H_{0}\right)}{H_{m}-H_{0}}
$$

one deduces from the second order expansions of magnitude in (A-5) and phase in (A-6) that the transmittance expressed in (14) can be simplified as:

$$
H(j \omega)=H_{0} e^{\frac{\tau_{0}^{2}\left(H_{m}+H_{0}\right)}{2\left(H_{m}-H_{0}\right)} \omega^{2}-j \tau_{0} \omega}
$$

With this expression in mind, one demonstrates that the output Fourier transform expressed in (A-2) becomes:

$$
Y(j \omega) \approx H_{0} \Delta T \sqrt{2 \pi} \cdot e^{-\left[\Delta T^{2}-\frac{\tau_{0}^{2}\left(H_{m}+H_{0}\right)}{2\left(H_{m}-H_{0}\right)}\right] \frac{\omega^{2}}{2}-j \omega\left(t_{0}-\tau_{0}\right)} .
$$

So, by taking:

$$
\Delta T_{1}=\sqrt{\Delta T^{2}-\frac{\tau_{0}^{2}\left(H_{m}+H_{0}\right)}{2\left(H_{m}-H_{0}\right)}} \Rightarrow \Delta T_{1}=\sqrt{\Delta T^{2}-\frac{L^{2}\left(1+g_{m} R_{d s}\right)\left[g_{m}\left(R+R_{d s}\right)-1\right]}{2\left(1-g_{m} R_{d s}\right)^{2}\left(R+R_{d s}\right)^{2}}}
$$

the previous expression output Fourier transform will be simplified as:

$$
Y(j \omega) \approx \Delta T \cdot H_{0} \cdot \sqrt{2 \pi} \cdot e^{-\frac{\left(\Delta T_{1} \omega\right)^{2}}{2}-j \omega\left(t_{0}-\tau_{0}\right)} .
$$

Then, by the inverse Fourier transform, it results the following time domain voltage response:

$$
y(t)=H_{0} \cdot \frac{\Delta T}{\Delta T_{1}} e^{-\frac{\left(t-t_{0}+\tau_{0}\right)^{2}}{2 \Delta T_{1}^{2}}} .
$$

Knowing that $H_{0}$ must be less than the maximal attainable gain, $H_{m}$, it should be noted that this output behaves as a Gaussian signal only if:

$$
\Delta T^{2}>\frac{\tau_{0}^{2}\left(H_{m}+H_{0}\right)}{2\left(1-H_{0}^{2}\right)\left(H_{m}-H_{0}\right)}>0,
$$

and with amplification if $H_{0} \cdot \Delta T / \Delta T_{1}>1$. One can see that this output signal is centered at the instant time $t-\tau_{0}$, thus, in advance of $\left|\tau_{0}\right|$ (because $\tau_{0}<0$ ) compared to the input expressed in (19) and presenting a standard deviation, $\Delta T_{1}$. This latter can be less the input one as explained by the following demonstration:

$$
\Delta T_{1}<\Delta T \Rightarrow R>0.5\left(1 / g_{m}-R_{d s}\right)
$$

In addition, under the condition: 


$$
\tau_{0} \ll \Delta T \sqrt{\frac{2\left(H_{m}-H_{0}\right)}{H_{m}+H_{0}}}
$$

one can consider that $\Delta T_{1} \approx \Delta T$. As stated by the expression below, this means that the output, $y(t)$ is closely equal to the input, $x(t)$ temporally shifted of $\tau_{0}$ with magnitude $H_{0}$-times more .

$$
y(t)=H_{0} e^{-\frac{\left(t-t_{0}+\tau_{0}\right)^{2}}{2 \Delta T_{1}^{2}}}=H_{0} x\left(t+\tau_{0}\right) .
$$

From this relation, it can be concluded that if $\tau_{0}<0$ (respectively $\tau_{0}>0$ ), the output is temporally in advance (respectively delayed) of $\left|\tau_{0}\right|$ and amplified by $H_{0}$. Nonetheless this theoretic explanation of the pulse advance, it is worth recalling that because of the drain-source current direction, the signs of the output and input voltages of a single cell are intrinsically unlike. Therefore, in practice, it leads us to use either two, or an even number of cascaded cells. This reason makes the smart thinking about the summary analysis of the identical two-stage of the proposed NGD cell. To concretize these theoretic findings, the next section will deal with the validation through simulations and experiments evidencing this NGD phenomenon both in frequency and time domains.

\section{EXPERIMENTAL INVESTIGATIONS}

The circuit designs and the simulations presented in this section were performed with the Advanced Design System $(A D S)$ electronic circuit simulator from Agilent ${ }^{T M}$. Prototypes with one- and two-stage NGD cells were designed, implemented and tested.

\subsection{Experimentation with single NGD cell}

Fig. 7(a) depicts the schematic of the fabricated one-stage NGD circuit. This circuit is fed in active load bias with power consumption $V_{g s}=0, V_{d s}=3 \mathrm{~V}$ and $I_{d s s}=110 \mathrm{~mA}$ by using the PHEMT ATF-34143 transistor manufactured by Avago Technology ${ }^{T M}$. After optimization, one extracts the following FET characteristics, $g_{m}=226 \mathrm{mS}$ and $R_{d s}=27 \Omega$. Afterward, synthesis and EM/circuit co-simulations were made with the Momentum and schematic environments of $A D S$. So that, the layout of the hybrid planar circuit formed by chip passive components $\mathrm{R}, \mathrm{L}$ and $\mathrm{C}$ respectively pictured in Fig. 7(b) was designed and implemented.

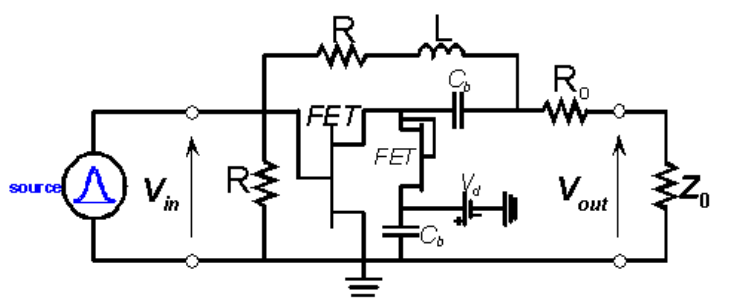

(a)

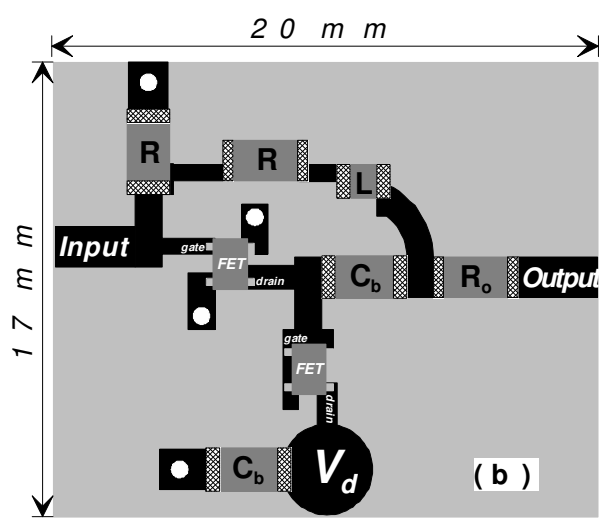

Fig. 7. (a) Detailed schematic and (b) layout of the tested NGD circuit with one-stage of NGD cell: $R=56 \Omega, R_{o}=10 \Omega, Z_{0}=50 \Omega, L=220 \mathrm{nH}, C_{b}=100 \mathrm{nF}$ using the FET ATF-34143 $\left(V_{d}=3 \mathrm{~V}, I_{d}=110 \mathrm{~mA}\right)$. 
This circuit is printed on the epoxy substrate FR4 with permittivity $\varepsilon_{r}=4.3$ and thickness $h=800 \mu \mathrm{m}$. Table 1 summarizes the characteristics of SMC passive components used during the simulations by considering their realistic Sparameter models proposed by the manufacturers and those implemented to the test device presented in Fig. 7(b).

\begin{tabular}{|c|c|c|c|}
\hline & Nominal value & Size & Reference \\
\hline $\boldsymbol{R}$ & $56 \Omega$ & \multirow{2}{*}{1206 [inch] } & \multirow{2}{*}{ WELWYN/PWC2010-XXRJI } \\
\hline $\boldsymbol{R}_{\boldsymbol{o}}$ & $10 \Omega$ & & \\
\hline $\boldsymbol{L}$ & $220 \mathrm{nH}$ & 0805 [inch] & $\begin{array}{l}\text { CoilCraft 0805CS-221XKBC, Q- } \\
\text { factor } 10 \% @ 100 \mathrm{MHz}\end{array}$ \\
\hline$C_{b}$ & $100 \mathrm{nF}$ & 1206 [inch] & SC KMT X7R 12065J \\
\hline
\end{tabular}

Table 1: Nominal values of the SMC passive components considered during the simulations and references of the components comprising the circuit shown in Figs. 7.

The next paragraphs reports the simulations and measurement results obtained with the prototype proposed both in frequency- and time-domains.

\subsubsection{Frequency-domain results}

To deploy the magnitude and the group delay of the fabricated circuit transfer parameter or insertion gain, one proceeds with the traditional RF-circuit frequency measurement with a vector network analyzer. Then, one considers the Sparameters of the tested circuit recorded experimentally which is defined as a 2 x 2 matrix.
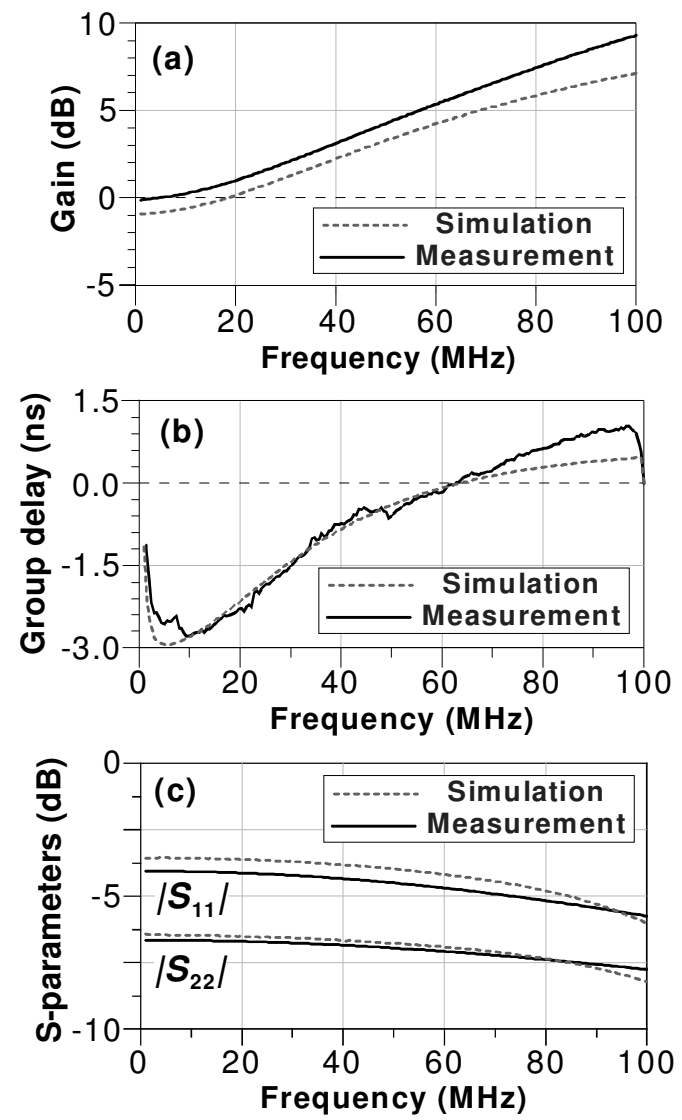

Fig. 8. Comparisons of the simulated and measured frequency responses: 
(a) gain, (b) group delay and (c) input/output parameters.

As illustrated in Fig. 8(a), a measured gain higher than $0 \mathrm{~dB}$ and slightly above the simulation mainly is obtained due to the imperfection of the used transistor non linear model provided by the manufacturer. As revealed in Fig. 8(b), the measured group delay is well-correlated with the simulation. Then, the access S-parameters of the circuit are presented in Fig. 8(c). As expected, this prototype of one-stage NGD circuit presented in Figs. 7 provides a baseband negative group delay. One observes that the tested circuit presents an NGD from DC up to $63 \mathrm{MHz}$ which can go down below $2.5 \mathrm{~ns}$.

\subsubsection{Time-domain simulations and measurements}

One points out that to perform simulations and experimentations results presented in this paper, the tested circuit was loaded by $Z_{0}=50 \Omega$.

To highlight the NGD effect in function of the input signal bandwidth, inputs with different time-widths or different frequency bands were generated. Hence, transient simulations run in ADS schematic environment with Gaussian pulse signal inputs presenting different arbitrary half-height time-widths $\left(T_{w}=\{2.5 \mathrm{~ns}, 7.5 \mathrm{~ns}, 11.6 \mathrm{~ns}\}\right)$ were injected in the test device. Therefore, one gets the normalized signals shown in Fig. 9. It can be seen that the output signal is more and more distorted when its width $T_{w}$ is shorter. This fact can be explained the most of input harmonic components are outside the NGD bandwidth then, they must propagate with positive group delay. Therefore, one finds here that the signal advance effect does not appear clearly as expected notably if $T_{w}<3 \mathrm{~ns}$.
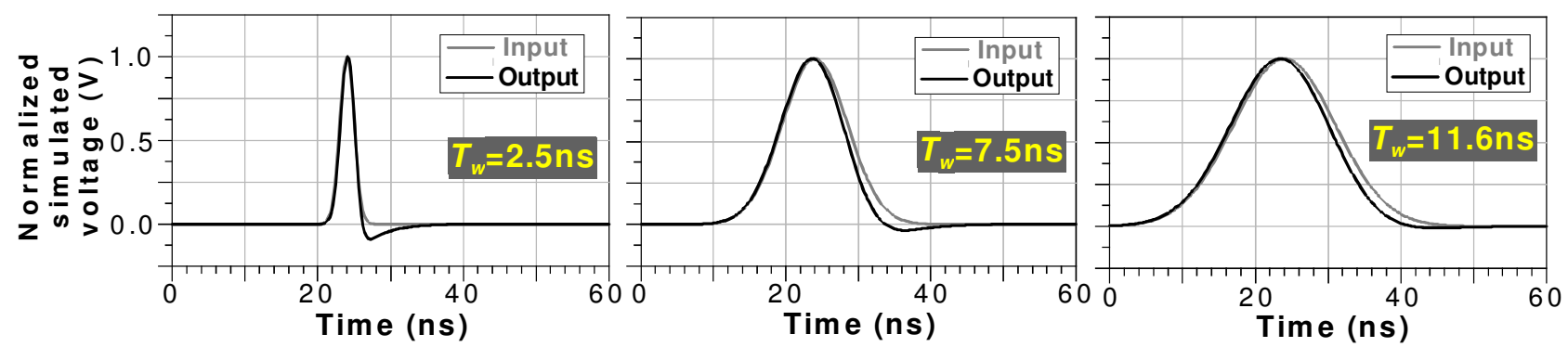

Fig. 9. Time-domain normalized simulation results with one-cell NGD circuit excited by Gaussian pulses having different widths (the opposite sign of the output is plotted).

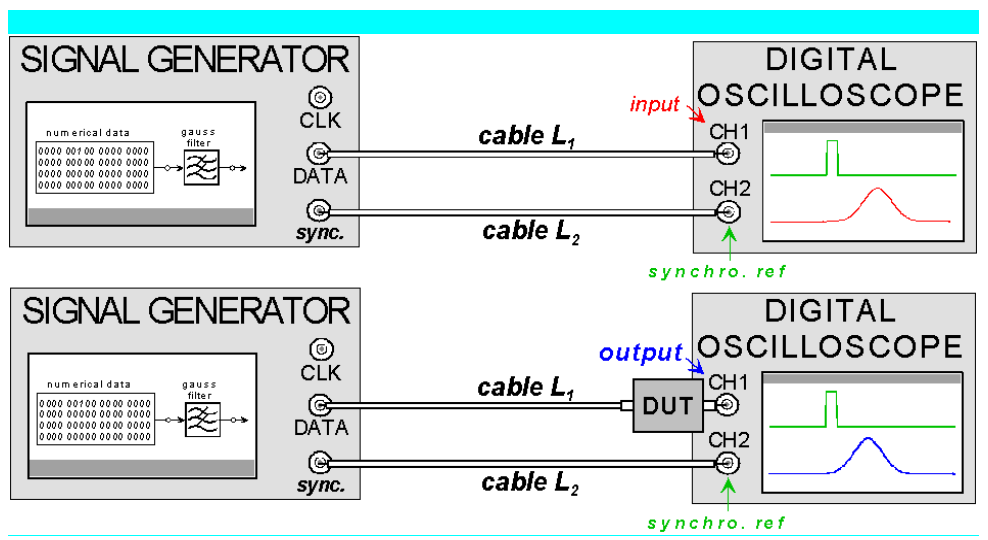

Fig. 10. Diagram of the experimental setup. 
To verify the feasibility of the NGD concept with UWB signals, the diagram presented in Fig. 10 were realized as an experimental setup. It consists to retrieve the input $(\mathrm{CH} 1)$ and output $(\mathrm{CH} 2)$ signals successively with the same reference of synchronization reference. As consequence, one gets the time domain results displayed in Fig. 11.

As observed in Fig. 11(a), the opposite of the plotted output voltage is slightly amplified compared to the input one. Moreover, according to the NGD frequency band in Fig. 8(b), this output (which can be assumed as a Gaussian pulse) with peak in advance of about $-1.5 \mathrm{~ns}$ compared to the input one was measured. In relative value, it corresponds approximately to $20 \%$ of its half-height time-width.

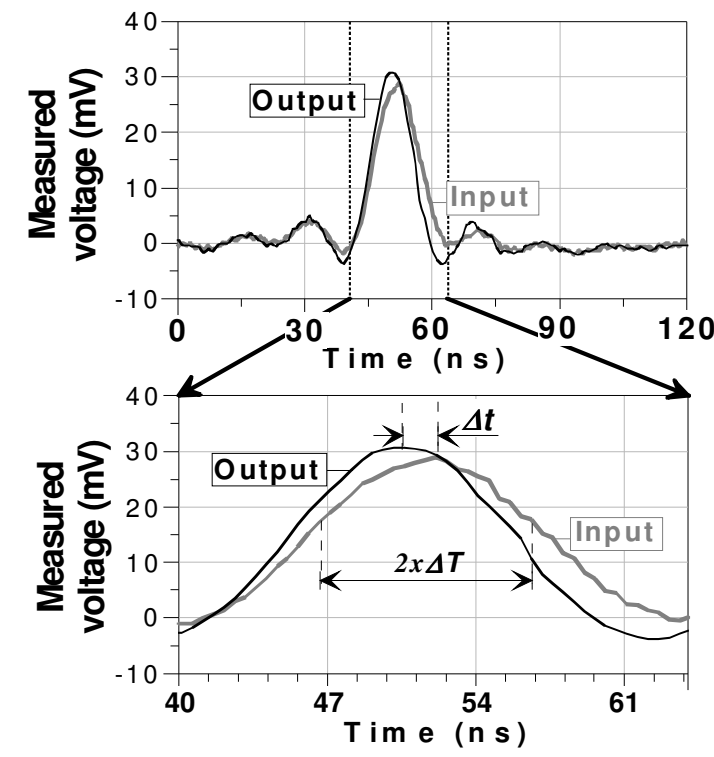

Fig. 11. Time-domain measured input and output (opposite sign) pulses.

Moreover, one points up that physically, it implies a negative group velocity of about $v_{g}=-0.13 \times c$, where $c$ is the vacuum light speed. As monitored by the zoom in graph of the bottom of Fig. 11, the leading and trailing edges of the obtained output pulse are equally in advance. This result reveals then, the apparition of negative frontal velocity corresponding to the signal section included in $10 \%$ and $90 \%$ of its maximal value. In fact, such an advance phenomenon can appear only when more than $95 \%$ of the input power spectrum density is included to the frequency band of the NGD. So that, a relatively smoothed input signal is necessary in order to realize such a time-advance because the signal presenting discontinuity (in time-domain) as a unit step Heaviside cannot propagate in negative delay compared to the input. Although this signal integrity conservation, it is worth noting that with one cell circuit, an output voltage cannot present the same polarity as the input. To overcome this effect, another prototype composed of two-stage NGD cell was designed and investigated in the next subsection.

\subsection{Experimentation with two-stage NGD cell}

Fig. 12 and Figs. 13 represent the RF-schematic of the understudy circuit and the hybrid device fabricated, which consists of two identical NGD cells cascaded. It was implemented with a low noise PHEMT ATF-55143 $\left(g_{m}=220 \mathrm{mS}\right.$, $R_{d s}=230 \Omega$ ). The input and output matching is guaranteed by inserting a series resistor, $R_{m}=33 \Omega$ at the input and the output of the whole circuit. 


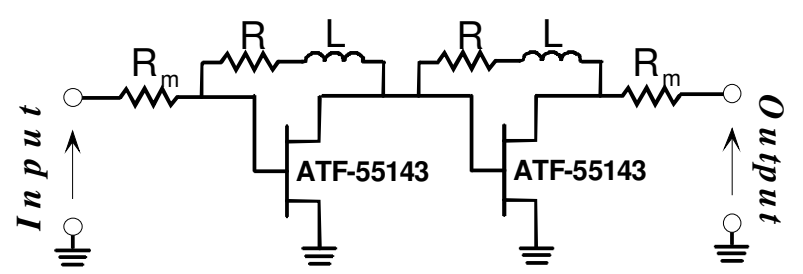

Fig. 12. Schematic of the two-stage active NGD circuit $\left(R=68 \Omega, R_{m}=33 \Omega, L=91 \mathrm{nH}\right)$.

It is worth noting that the matching series resistances, $R_{m}$ are added here for the test two-stage circuit because they allow to correct considerably the inter-stage reflected signals in the operated frequency band. The layout shown in Fig. 13(a) was printed on an FR4 substrate with permittivity, $\varepsilon_{r}=4.3$, and thickness, $h=800 \mu \mathrm{m}$. Simulations were first performed by using the simplified FET model and the ADS realistic S-parameter models of lumped components listed in Table 2.
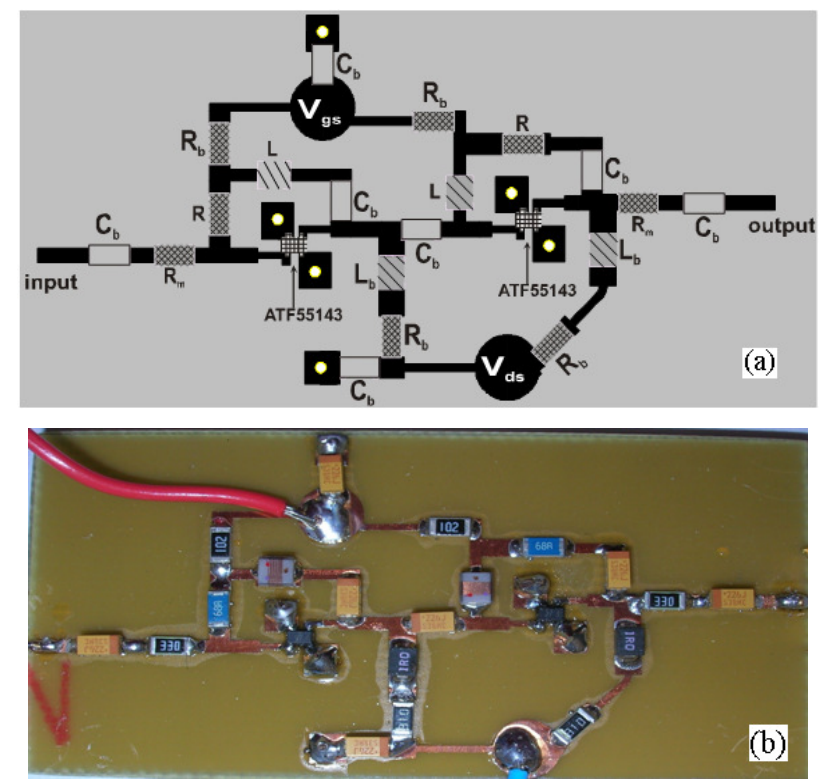

Fig. 13. (a) Layout and (b) photograph of the realized circuit $(57.6 \mathrm{~mm} \times 29.4 \mathrm{~mm})$ including biasing network $\left(R_{b}=1 \mathrm{k} \Omega, C_{b}=22 \mu \mathrm{F}, L_{b}=1 \mu \mathrm{H}\right)$.

To simulate this two-stage NGD device, S-parameter models of the components proposed by the manufacturers combined with the EM-model of the layout including the metal interconnect and substrate effects were considered. Table 2 summarizes the nominal values of the SMC component S-parameters used during the simulations and references of those implemented in Fig. 14.

\begin{tabular}{|c|c|c|c|}
\hline & Nominal value & Size & References \\
\hline$R$ & $68 \Omega$ & 1206 [inch] & \multirow{3}{*}{ WELWYN/PWC2010-XXRJI } \\
\hline $\boldsymbol{R}_{m}$ & $33 \Omega$ & 1206 [inch] & \\
\hline $\boldsymbol{R}_{b}$ & $1 \mathrm{k} \Omega$ & 1206 [inch] & \\
\hline$L$ & $270 \mathrm{nH}$ & 0805 [inch] & $\begin{array}{c}36500805 \text { 91N 5\% TYCO } \\
\text { ELECTRONICS }\end{array}$ \\
\hline
\end{tabular}




\begin{tabular}{c|c|c|c}
\hline$L_{b}$ & $1 \mu \mathrm{H}$ & $1812[$ inch] & $\begin{array}{c}\text { 3613C1R0K TYCO } \\
\text { ELECTRONICS }\end{array}$ \\
\hline$C_{b}$ & $22 \mu \mathrm{F}$ & $1206[$ inch] & $\begin{array}{c}\text { PHYCOMP } \\
\text { CC1206ZKY5V6BB226 }\end{array}$ \\
\hline
\end{tabular}

Table 2: References of the SMC lumped elements constituting the tested circuit presented in Figs. 13.

\subsubsection{Frequency-domain results}

After EM/circuit co-simulations, the gain displayed in Fig. 14(a) and the group delay depicted in Fig. 14(b) which are plotted in dashed curves are obtained. As forecasted in theory, once again, baseband negative group delay accompanied by voltage amplification is obtained. Moreover, the simulations are in good agreement with the measurements.
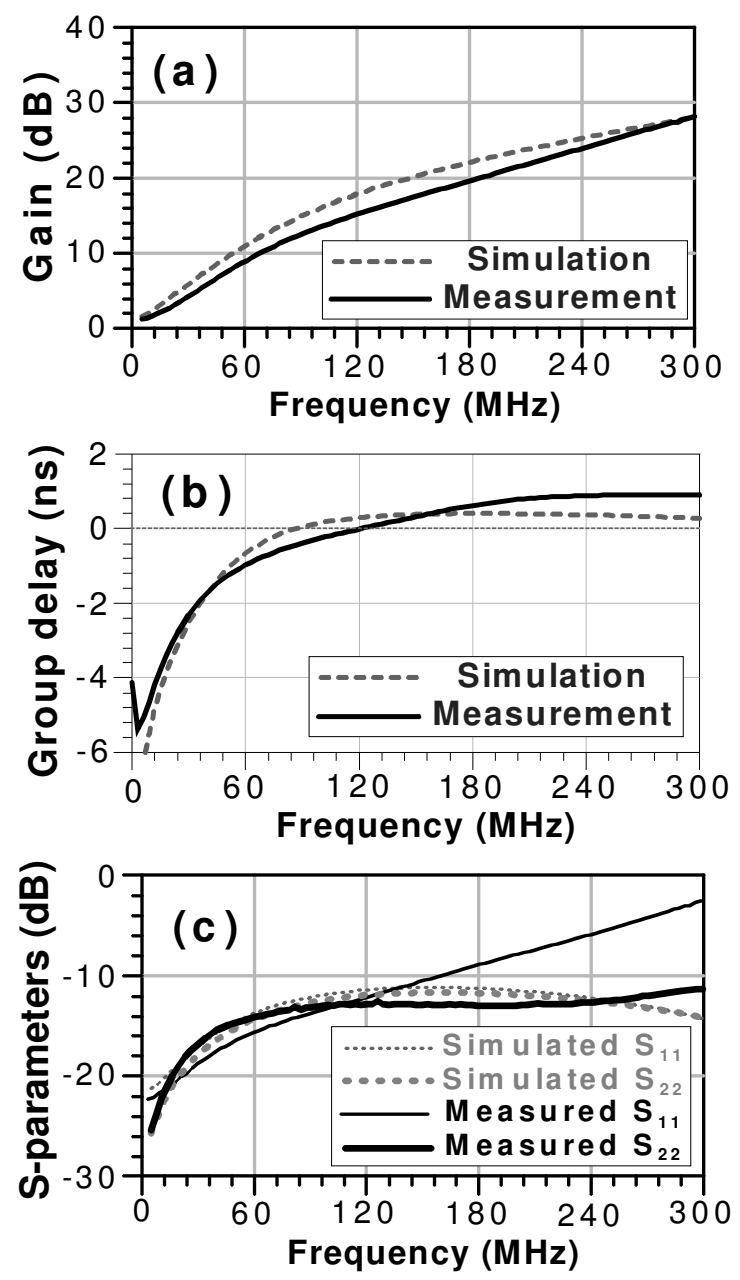

Fig. 14. Comparison of the simulated and measured frequency responses of the fabricated device presented in

Figs. 13: (a) gain, (b) group delay and (c) input/output parameters.

It shows that the group delay is negative up to about $100 \mathrm{MHz}$ with a minimum gain of $0.45 \mathrm{~dB}$. After retro-simulation of the tested circuit by taking into account the non-linear-model of the employed FET ATF-55143 biased at $V_{d s}=3 \mathrm{~V}$, $I_{d s}=52 \mathrm{~mA}$, it can be seen that the measured and simulated gains are relatively well-fitted. The slight differences here are mainly due to the undesired parasitic effects of the FET and the dispersion of the lumped SMCs at hundreds MHz.

\subsubsection{Time-domain simulations and measurements}


Similar to the previous subsection, simulations with typically Gaussian pulses with different half height time-width denoted $T_{w}$ were carried out. As depicted by the normalized simulation voltages shown in Fig. 15, due to the excessive bandwidth of the input signal, the output can be significantly distorted when the input time-width is very short or inversely, the input signal spectrum is very wide in frequency domain.
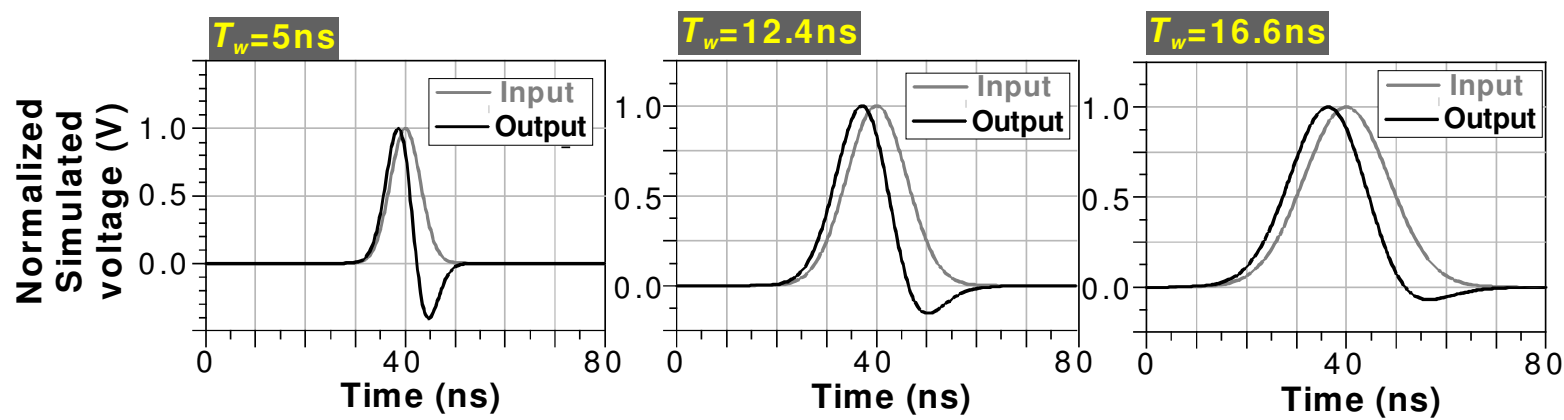

Fig. 15. Time-domain normalized simulation results with two-cell NGD circuit excited by Gaussian pulses having different widths.

The measurement results reported in this paragraph were generated by using a UWB Gaussian wave input pulse with a half-height width of about $17 \mathrm{~ns}$. To record the measured signals, the input and output circuit accesses were directly connected to the scope through a coaxial tee with no use of cable to avoid additional delays. In fact, it enables to demonstrate undeniably the existence and the apparition of the time-advance phenomenon through a simultaneous visualization of the input and output. So, the recorded signals cumulate the transition delays introduced by the input and output tees (approximately of about $1 \mathrm{~ns}$ with no NGD circuit) which was de-embedded from rough measurements prior to the production of Fig. 16. By comparison with the input pulse, as predicted in theory, the measured output behaves as a Gaussian pulse fairly amplified, presenting a peak absolute advance of about $5 \mathrm{~ns}$. This graph confirms undeniably the verification of the negative signal delay thanks to the NGD function with an ultra-short transient signal.

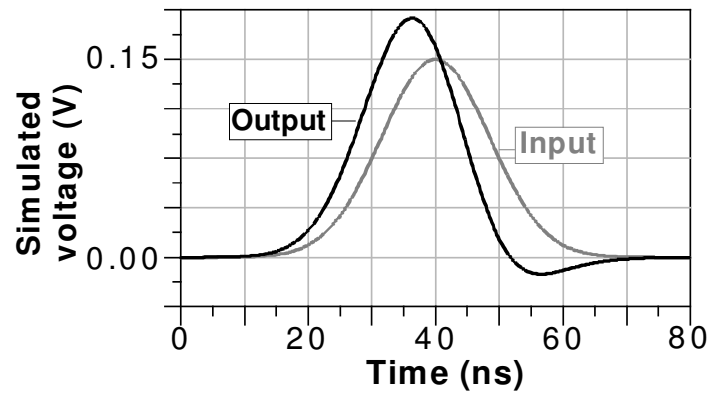

(a)

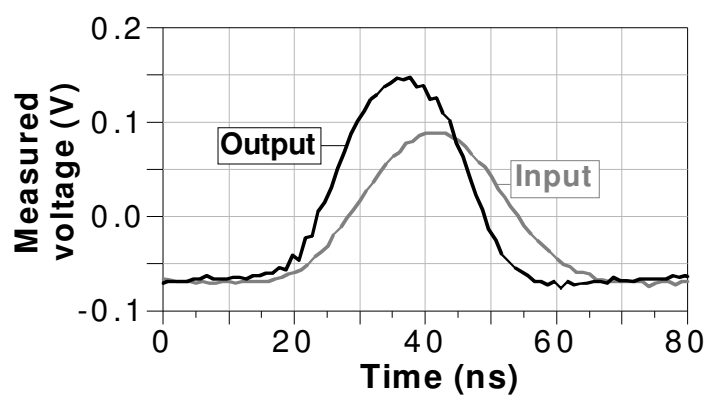

(b)

Fig. 16. Time-domain results: (a) simulations and (b) measurements.

One evaluates that it corresponds to $31 \%$ of its half-height width with respect to the input standard deviation. Moreover, in physical point of view, it raises a negative group velocity of about $v_{g}=-0.12 \times c$. As aforementioned in subsection 2.4, one observes that the transient output pulse width is slightly compressed compared to the input one. Furthermore, it can be seen clearly that this negative signal delay is accompanied by the time-advance of up and down output front edges. Such an effect was realized because as displayed in Fig. 14(b), more than $95 \%$ of the input energy 
spectrum is including in the NGD frequency band.

Despite these results, it is important to note that as argued in [23-29], this time advance phenomenon does not forbid the signal causality.

\section{CONCLUSIONS}

Theoretic, numerical and experimental demonstrations of the possibility to generate an ultra-short duration transient signal with negative delay are successfully investigated by using an NGD active circuit. This innovative circuit is composed of an RL-series network in feedback with a FET.

It was pointed up by many research works that with NGD passive devices [30-33], the apparition of the NGD is generally accompanied with excessive losses in the operating frequency band. To compensate the losses, an active electronic topology is herein deeply investigated. So, it was demonstrated that the introduced active circuit is susceptible to exhibit an UWB NGD in baseband frequency up to microwave frequencies simultaneously with amplification. Theoretic approaches illustrating in detail the fundamental principle of this outstanding phenomenon were offered and validated numerically and experimentally. The basic characteristics and properties of the understudy NGD cell are established. Contrarily to the classical electronic passive device, the NGD effect becomes more and more important when the cascaded NGD cell number increases. This statement was verified experimentally with two prototypes of simple electronic devices implemented in planar hybrid technology. As results, frequency responses and time domain signals from measurements confirm the efficiency of the theoretical prediction. Then, it was observed visually a possibility to propagate output pulse peak and fronts advances of UWB transient pulse. One points up that to realize such a phenomenon; the main rule is that the output pulse can be in advance compared to its input one when this last one presents $95 \%$ of its density signal spectrum included in the frequency band of the NGD, no matter its origin. Other than this NGD function, it is shown that this circuit can generate also a pulse compression in time domain.

As future works, the analysis of the noise contribution in this type of NGD cell is in progress. Nonetheless the frequency and time domain proofs, dealing with the apparition of the time advance, the interpretation of the information velocity facing to the NGD aspect remains an open question. To draw attention to this scientist debate, more convincing results have been planned in the continuation of this study.

\section{APPENDIX A}

It is well-known that the Fourier transform of the Gaussian signal expressed in (19) of subsection 2.4 is:

$$
X(j \omega)=\Delta T \sqrt{2 \pi} \cdot e^{-(\Delta T \omega)^{2} / 2-j \omega t_{0}} .
$$

Knowing that $H(j \omega)=e^{\ln |H(j \omega)|} . e^{j \varphi(\omega)}$, one can write the output Fourier transform:

$$
Y(j \omega)=\Delta T \sqrt{2 \pi} \cdot e^{-(\Delta T \omega)^{2} / 2-j \omega t_{0}} \cdot e^{0.5 \ln [H(j \omega)]+j \varphi(\omega)},
$$

where the gain, $|H(j \omega)|$ and phase, $\varphi(\omega)$ are given by:

$$
\begin{aligned}
& |H(j \omega)|=H_{0} \sqrt{\frac{\left(H_{0}-H_{m}\right)^{2}+\left(\omega \tau_{0} H_{m}\right)^{2}}{\left(H_{0}-H_{m}\right)^{2}+\left(\omega \tau_{0} H_{0}\right)^{2}}}, \\
& \varphi(\omega)=\arctan \left[\frac{\omega \tau_{0}\left(H_{0}-H_{m}\right)^{2}}{H_{0} H_{m}\left(\omega \tau_{0}\right)^{2}+\left(H_{0}-H_{m}\right)^{2}}\right] .
\end{aligned}
$$


By denoting respectively, $F^{\prime}(0)=\left.\frac{\partial F(\omega)}{\partial \omega}\right|_{\omega=0}$ and $F^{\prime \prime}(0)=\left.\frac{\partial^{2} F(\omega)}{\partial \omega^{2}}\right|_{\omega=0}$, the first- and the second- order derivative of the function, $F(\omega)$ with respect to $\omega$, one can write the second order MacLaurin series expansion of (A-3) and (A-4) as:

$$
\begin{gathered}
\ln |H(j \omega)| \approx \ln |H(0)|+\frac{H^{\prime}(0)}{H(0)} \omega+\frac{1}{2} \frac{H^{\prime \prime}(0)}{H(0)} \omega^{2}+O\left(\omega^{2}\right) \approx \ln \left|H_{0}\right|+\frac{\tau_{0}^{2}\left(H_{m}+H_{0}\right)}{2\left(H_{m}-H_{0}\right)} \omega^{2}+O\left(\omega^{2}\right), \\
\varphi(\omega) \approx \varphi(0)+\varphi^{\prime}(0) \omega+\frac{1}{2} \varphi^{\prime \prime}(0) \omega^{2}+O\left(\omega^{2}\right)=\tau_{0} \omega+O\left(\omega^{2}\right)
\end{gathered}
$$

\section{REFERENCES}

[1] H. Lamb, “On Group-Velocity”, Proc. London Math. Soc. 1, pp. 473-479, 1904.

[2] A. Schuster, "An Introduction to the Theory of Optics", Edward Arnold, London, pp. 313-318, 1904.

[3] A. Sommerfeld, "Ein Einwand gegen die Relativtheorie der Elektrodynamik und seine Beseitigung", Physik. Z. 8, pp. 841-842, 1907.

[4] A. Sommerfeld, "Uber die Fortpflanzung des Lichtes in Dispergierenden Medien”, Ann. Physik. 44 (in German), pp. 177-201, 1914.

[5] L. Brillouin, "Uber die Fortpflanzung des Lichtes in Dispergierenden Medien”, Ann. Physik. 44 (in German), pp. 203-240, 1914.

[6] L. Brillouin, "Wave Propagation in Periodic Structures", McGraw-Hill, New York, 1946.

[7] A. Sommerfeld, "Lectures on Theoretical Physics", Optics. Academic Press Inc. US, 1954.

[8] L. Brillouin, "Wave Propagation and Group Velocity”, Academic Press, New York, pp. 1-83 \& 113-137, 1960.

[9] R. Y. Chiao, "Superluminal (but Causal) Propagation of Wave Packets in Transparent Media with Inverted Atomic Populations", Phys. Rev. A, Vol. 48, pp. R34-R37, 1993.

[10] L. J. Wang, A. Kuzmich and A. Dogariu, "Gain-Assisted Superluminal Light Propagation”, Nature 406, pp. 277 $279,2000$.

[11] O. F. Siddiqui, M. Mojahedi and G. V. Eleftheriades, "Periodically Loaded Transmission Line with Effective Negative Refractive Index and Negative Group Velocity", IEEE Trans. Ant. Prop., Vol. 51, No. 10, pp. 2619-2625, Oct. 2003.

[12] J. F. Woodley and M. Mojahedi, "Negative Group Velocity and Group Delay in Left-Handed Media", Phys. Rev. E, Vol. 70, pp. 046603.1-046603.6, 2004.

[13] J. C.-E. Sten and A. Hujanen, "Aspects on the phase delay and phase velocity in the electromagnetic near-field," Progress In Electromagnetics Research, Vol. 56, pp. 67-80, 2006.

[14] J. Mobley, "The Time-Domain Signature of Negative Acoustic Group Velocity in Microsphere Suspensions," J. Acoust. Soc. Am., Vol. 122, No. 1, EL8-EL14, Jul. 2007.

[15] W. M. Robertson, J. Pappafotis, P. Flannigan, J. Cathey, B. Cathey and C. Klaus, "Sound Beyond the Speed of Light : Measurement of Negative Group Velocity in an Acoustic Loop Filter”, App. Phys. Lett., Vol. 90, No. 1, pp. 014102.1-014102.3, 2007.

[16] B. Macke and B. Segard, "Two-Pulse Interference and Superluminality," Optics Communications, Vol. 281, No. 1, pp. 12-17, 2008. 
[17] A. Dogariu, A. Kuzmich and L. J. Wang, "Transparent Anomalous Dispersion and Superluminal Light-Pulse Propagation at a Negative Group Velocity,” Phys. Rev. A, Vol. 63, pp. 053806.1-053806.12, 2001.

[18] D. J. Gauthier and R. W. Boyd, "Fast Light, Slow Light and Optical Precursors: What Does It All Mean", Photonics Spectra, pp. 82-90, Jan. 2007.

[19] C. G. B. Garrett and D. E. McGumber, "Propagation of a Gaussian Light Pulse through an Anomalous Dispersion Medium”, Phys. Rev. A, Vol. 1, pp. 305-313, 1970.

[20] S. Chu and S. Wong, "Linear Pulse Propagation in an Absorbing Medium”, Phys. Rev. Lett., Vol. 48, pp. 738-741, 1982.

[21] B. Ségard and B. Macke, “Observation of Negative Velocity Pulse Propagation”, Phys. Lett. 109, pp. 213-216, 1985 .

[22] J. N. Munday and W. M. Robertson, "Observation of Negative Group Delays within a Coaxial Photonic Crystal Using an Impulse Response Method” Optics Communications, Vol. 273, Issue 1, pp. 32-36, 2007.

[23] R. Y. Chiao, E. L. Bolda, J. Bowie, J. Boyce and M. W. Mitchell, "Superluminality and Amplifiers", Prog. Crystal Growth Charact. Mat. 33, pp. 319-325, 1996.

[24] M. W. Mitchell and R.Y. Chiao, “Causality and Negative Group Delays in a Simple Bandpass Amplifier”, Am. J. Phys., Vol. 66, pp. 14-19, 1998.

[25] M. W. Mitchell and R.Y. Chiao, "Negative Group Delay and 'Fronts' in a Causal Systems: An Experiment with Very Low Frequency Bandpass Amplifiers”, Phys. Lett. A, Vol. 230, pp. 133-138, Jun. 1997.

[26] M. Kitano, T. Nakanishi and K. Sugiyama, "Negative Group Delay and Superluminal Propagation: An Electronic Circuit Approach”, IEEE J. Sel. Top. in Quantum Electron., Vol. 9, No. 1, pp. 43-51, Feb. 2003.

[27] T. Nakanishi, K. Sugiyama and M. Kitano, "Demonstration of Negative Group Delays in a Simple Electronic Circuit”, Am. J. Phys., Vol. 70, Issue 11, pp. 1117-1121, 2002.

[28] J. N. Munday and R. H. Henderson, "Superluminal Time Advance of a Complex Audio Signal”, Appl. Phys. Lett., Vol. 85, pp. 503-504, Jul. 2004.

[29] D. Solli, R. Y. Chiao and J. M. Hickmannn,, "Superluminal Effects and Negative Group Delays in Electronics, and their Applications", Phys. Rev. E, Vol. 66, pp. 056601.1-056601.4, 2002.

[30] S. Lucyszyn, I. D. Robertson and A. H. Aghvami, "Negative Group Delay Synthesiser”, Electron. Lett., Vol. 29, pp. 798-800, 1993.

[31] C. D. Broomfield and J .K. A. Everard, "Broadband Negative Group Delay Networks for Compensation of Oscillators, Filters and Communication Systems”, Electron. Lett., Vol. 23, pp. 1931-1933, Nov. 2000.

[32] G. V. Eleftheriades, O. Siddiqui and A. K. Iyer, "Transmission Line for Negative Refractive Index Media and Associated Implementations without Excess Resonators”, IEEE MWC Lett., Vol. 13, No. 2, 51, pp. 51-53, Feb. 2003.

[33] O. F. Siddiqui, S. J. Erickson, G. V. Eleftheriades, and M. Mojahedi, "Time-Domain Measurement of NegativeIndex Transmission-Line Metamaterials”, IEEE Trans. MTT, Vol. 52, No. 5, pp. 1449-1453, May 2004.

[34] B. Ravelo, A. Perennec, M. Le Roy and Y. Boucher, "Active Microwave Circuit with Negative Group Delay," IEEE MWC Lett., Vol. 17, Issue 12, pp. 861-863, Dec. 2007.

[35] B. Ravelo, A. Perennec and M. Le Roy, "Synthesis of Broadband Negative Group Delay Active Circuits," IEEE MTT-S Int. Symp. Dig., pp. 2177-2180, June 2007.

[36] B. Ravelo, A. Perennec and M. Le Roy, "Negative Group Delay Active Topologies Respectively Dedicated to Microwave Frequencies and Baseband Signals,” J. EuMA, Vol. 4, Issue 2, pp. 124-130, Jun. 2008. 
[37] B. Ravelo, A. Perennec and M. Le Roy, "Equalization of Interconnect Propagation Delay with Negative Group Delay Active Circuits”, Proc. of 11th IEEE Workshop SPI, Genova, Italy, pp. 15-18, May 2007.

[38] B. Ravelo, A. Perennec and M. Le Roy, "Application of Negative Group Delay Active Circuits to Reduce the 50\% Propagation Delay of RC-Line Model”, Proc. of 12th IEEE Workshop SPI, Avignon, France, May 2008.

[39] B. Ravelo, A. Perennec and M. Le Roy, "Experimental Validation of the RC-Interconnect Effect Equalization with Negative Group Delay Active Circuit in Planar Hybrid Technology,” Proc. of 13th IEEE Workshop SPI, Strasbourg, France, May 2009.

[40] B. Ravelo, A. Perennec and M. Le Roy, "New Technique of Inter-Chip Interconnect Effects Equalization with Negative Group Delay Active Circuits,” VLSI, Chap. 20, INTECH Book edited by: Zhongfeng Wang, Feb. 2010 , pp. 409-434.

[41] V. Adler and E. G. Friedman, "Repeater Design to Reduce Delay and Power in Resistive Interconnect", IEEE TCAS II, Analog and Digital Signal Processing, Vol. 54, No. 5, pp. 607-616, May 1998.

[42] M. Bartolini, P. Pulici, P. P. Stoppino and G. Campardo, “A Reduced Output Ringing CMOS Buffer,” IEEE TCAS II, Exp. Briefs, Vol. 54, No. 2, pp. 102-106, Feb. 2007.

[43] F. R. Awwad, M. Nekili, V. Ramachandran and M. Sawan, "On Modeling of Parallel Repeater-Insertion Methodologies for SoC Interconnects”, IEEE TCAS I, Vol. 55, No. 1, pp. 322-336, Feb. 2008.

[44] B. Ravelo, M. Le Roy and A. Perennec, “Application of Negative Group Delay Active Circuits to the Design of Broadband and Constant Phase Shifters," Microwave and Optical Technology Letters, Vol. 50, No. 12, pp. 3078-3080, Dec. 2008.

[45] B. Ravelo, A. Perennec and M. Le Roy, "Synthesis of frequency-independent phase shifters using negative group delay active circuit”, Int J. RF and Microwave Computer-Aided Engineering (RFMiCAE), Vol. 21, Issue 1, pp. 17-24, Jan. 2011. 